Final Progress Report (July 1, 2002 - June 30, 2006)

DOE Award Number: DE-FG07-02ID14332, Univ. of Michigan Project \#: F006740

\title{
Fast Neutron Imaging Systems
}

\author{
Zhong He \\ Department of Nuclear Engineering and Radiological Sciences \\ The University of Michigan, Ann Arbor, Michigan 48109-2104
}

During the final year of this project, our research focus has shifted from detecting fast neutrons using various 3-dimensional position-sensing gas detectors to 3-dimensional position-sensitive solid state detectors. In particular, extensive investigation had been carried out on 3-D CdZnTe detectors for detection of fission neutrons. Since elastic scattering between fast neutrons and nuclei in the detector is the most likely interaction, the logical approach is to develop a detection scheme based on elastic collisions between fast neutrons and detection material. Three-dimensional position-sensitive CdZnTe detectors have been developed by our group for gamma-ray imaging and spectroscopy operating at ambient temperatures. Their potential for compact hand-held instruments for nuclear non-proliferation and homeland security has been well demonstrated with excellent energy resolution, about 1\% FWHM at $662 \mathrm{keV}$ gamma-ray energy, and realtime gamma-ray imaging capability with angular resolution about 15 degrees on a single $1.5 \times 1.5 \times 1 \mathrm{~cm}^{3}$ detector. The detection of thermal neutrons using CdZnTe detectors was demonstrated since the middle of 1990s by Dr. Douglas McGreger at the University of Michigan. More than $90 \%$ of incoming thermal neutrons can be absorbed within the top $0.65 \mathrm{~mm}$ of CdZnTe due to a very large neutron capture cross-section of ${ }^{113} \mathrm{Cd}$. After the capture of thermal neutrons, gamma rays of characteristic energies, $558.6 \mathrm{keV}(72.7 \%)$, $575.8 \mathrm{keV}(2.3 \%)$, or $651.3 \mathrm{keV}(13.9 \%)$, will be emitted that can be recorded as signatures of thermal neutrons by CdZnTe detectors. However, the detection of fast neutrons using high-Z semiconductor detectors has never been attempted. The key reason is that the energy transfer between a fast neutron and a nucleus with an atomic number around 50 by elastic scattering is very small, below the low-energy threshold of most solid state detectors.

Because the inherent potential to achieve very low energy threshold on 3dimensional position-sensitive CdZnTe detectors, we studied the possibility of detecting fast neutrons using 3-D CdZnTe detectors. The detection concept is to recognize multiple low-energy depositions within the bulk of 3-dimensional position-sensitive CdZnTe detectors, as the signature of fission neutrons. When gamma rays interact with CdZnTe, low energy gammas deposit their energy in a single location through photoelectric interaction, thus can be clearly distinguished from multiple-site fast neutron events. High energy gamma rays (above $1.022 \mathrm{MeV}$ ) can interact with detector by pair production which leave electron-positron tracks with energy deposition much higher than the threshold for neutron detection. Intermediate-energy gammas can undergo multiple Compton scatterings, cause multiple low-energy depositions within the detector. Unfortunately, the probability of these events is very low, 2 to 3 orders of magnitude 
lower than that caused by fission neutrons. A systematic study had been performed using Monte-Carlo simulations, with measured detector properties, such as energy resolution, electronic noise and detection efficiency. Our simulation was based on the following facts: If we use one of the Polaris II 3-D CdZnTe detector system, which consists of two layers of three by three $2 \times 2 \times 1.5 \mathrm{~cm}^{3} \mathrm{CdZnTe}$ modular detectors (with a total detection volume of $108 \mathrm{~cm}^{3}$ ), and the low-energy threshold could reach $1 \mathrm{keV}$, a 2-kg weapon grade $\mathrm{Pu}$ (containing $6 \%$ of ${ }^{240} \mathrm{Pu}$ by weight) could be detected with high confidence (about 91\% probability) in one minute. This low-energy threshold of $1 \mathrm{keV}$ is substantially lower than what can be achieved today with current technology. The electronic noise of our current ASIC electronic readout system VAS_UM1/TAT4 is about $3 \mathrm{keV}$. In order to reach $1 \mathrm{keV}$ energy threshold, more development will be needed to reduce electronic noise of ASIC systems, and moderate cooling may be required for fission neutron detection.

Publications during the final year of the project (July 2005 - June 2006)

S.D. Kiff, Z. He, G. Tepper "Radial Position Sensing in a Coplanar-Grid High-Pressure Xenon Gamma-Ray Spectrometer," IEEE Transactions on Nuclear Science, Vol. 53, No.3 (2006) 1380 - 1384.

Z. He and S.D. Kiff "Detection of fission neutrons using 3-dimensional position-sensitive CdZnTe detectors," to be submitted to Nuclear Instruments and Methods in Physics Research A, 2006.

Scott D. Kiff will graduate with a Ph.D by the end of December 2006. His Ph.D thesis will be available from the University of Michigan Library after January 1, 2007.

Annual reports during the first three years of this project were submitted to DOE. They are available upon request. 